09

\title{
Электрическая площадь предельно коротких импульсов и момент силы
}

\author{
(C) Н.Н. Розанов $1,2,3$ \\ ${ }^{1}$ Государственный оптический институт им. С.И. Вавилова, \\ 199053 Санкт-Петербург, Россия \\ ${ }^{2}$ Университет ИТМО, \\ 197101 Санкт-Петербург, Россия \\ ${ }^{3}$ Физико-технический институт имени А.Ф. Иофрфе, \\ 194021 Санкт-Петербург, Россия \\ e-mail: nnrosanov@mail.ru
}

Поступила в редакцию 13.08.2018 г.

\begin{abstract}
Электрическая площадь импульса излучения, определяемая как интеграл от напряженности электрического поля по времени, интерпретируется как механический импульс силы Лоренца, действующей на единичный электрический заряд при его взаимодействии с импульсом излучения. Продемонстрировано соответствие между законом сохранения импульса классической механики и изменением импульса при взаимодействии квантового объекта с интенсивным предельно коротким импульсом излучения. Подтверждена высокая эффективность ускорения заряженных частиц с помощью квазиуниполярных импульсов излучения.
\end{abstract}

DOI: $10.21883 /$ OS.2018.12.46944.234-18

Электрическая площадь электромагнитных, в том числе оптических импульсов, обладает интересным свойством сохранения при распространении в макроскопических средах с диссипацией [1-6], которое описывается уравнениями Максвелла электродинамики сплошных сред [7] (из рассмотрения исключаются системы с запуском непрекращающейся генерации излучения). С другой стороны, такая площадь существенно определяет взаимодействие предельно коротких импульсов с квантовыми объектами [8] (ряд приведенных в [8] результатов ранее был получен в работе [9] и цитируемой там литературе). Электрическая площадь импульса определяется соотношением

$$
\mathbf{S}_{E}=\int_{t=-\infty}^{+\infty} \mathbf{E}(t) d t
$$

Здесь $\mathbf{E}-$ напряженность электрического поля и $t-$ время. Площадь $\mathbf{S}_{E}$ совпадает с механическим моментом $\mathbf{N}=\int_{-\infty}^{+\infty} \mathbf{F} d t$ силы Лоренца $\mathbf{F}=q \mathbf{E}$, действующей на единичный электрический заряд $q=1$; предполагается, что сила прикладывается в течение ограниченного времени и движение нерелятивистское, так что магнитной составляющей силы Лоренца можно пренебречь. Прямым следствием 2-го закона Ньютона $d \mathbf{p} / d t=F(t)$ [10], где $\mathbf{p}$ - механический импульс классической частицы, служит закон сохранения импульса

$$
\mathbf{p}-\mathbf{p}_{0}=\mathbf{N}
$$

где $\mathbf{p}_{0}-$ начальный импульс частицы (до начала действия силы). Задачей данного сообщения служит формулировка квантового аналога этого соотношения на простом примере взаимодействия квантовой частицы с предельно коротким и интенсивным импульсом излучения $[8,9]$.

Оператор и среднее значение импульса квантовой частицы с волновой функцией $\psi(\mathbf{r}, t)$ имеют соответственно вид [11]

$$
\hat{\mathbf{p}}=-i \nabla, \quad\langle\mathbf{p}\rangle=\langle\psi|\mathbf{p}| \psi\rangle=-i \int \psi^{*} \nabla \psi d \mathbf{r}
$$

Здесь $\mathbf{r}$ - радиус-вектор и $\nabla-$ оператор градиента; используется атомная система единиц и волновая функция считается нормированной. До начала действия импульса излучения волновая функция $\psi=\psi_{0}$ и среднее значение механического импульса

$$
\left\langle\mathbf{p}_{0}\right\rangle=\left\langle\psi_{0}|\mathbf{p}| \psi_{0}\right\rangle=-i \int \psi_{0}^{*} \nabla \psi_{0} d \mathbf{r} .
$$

Если начальное состояние отвечает стационарному состоянию дискретного спектра, то среднее значение импульса $\left\langle\mathbf{p}_{0}\right\rangle=0$. В указанных в [8] приближениях (длительность импульса излучения много меньше обратной частоты переходов в невозмущенной излучением системе, а его амплитуда превышает напряженность внутриатомного поля) волновая функция после окончания импульса излучения имеет вид произведения начальной волновой функции $\psi_{0}$ и волновой функции плоской волны с импульсом $-\mathbf{S}_{E}$ :

$$
\psi=\exp \left(-i \mathbf{S}_{E} \mathbf{r}\right) \psi_{0}
$$

Тогда среднее значение механического импульса частицы с отрицательным (единичным по модулю) электрическим зарядом после окончания действия импульса 
излучения

$$
\begin{aligned}
\langle\mathbf{p}\rangle= & -i \int \psi_{0}^{*}(\mathbf{r})\left[-i \mathbf{S}_{E} \psi_{0}(\mathbf{r})\right. \\
& \left.+\nabla \psi_{0}(\mathbf{r})\right] d \mathbf{r}=-\mathbf{S}_{E} \int\left|\psi_{0}(\mathbf{r})\right|^{2} d \mathbf{r} \\
& -i \int \psi_{0}^{*}(\mathbf{r}) \nabla \psi_{0}(\mathbf{r}) d \mathbf{r}=\left\langle\mathbf{p}_{0}\right\rangle-\mathbf{S}_{E}
\end{aligned}
$$

Это соотношение с учетом отрицательности электрического заряда частицы и служит квантовым аналогом классического закона сохранения импульса (2). Оно подтверждает также эффективность ускорения заряженных частиц с помощью квазиуниполярных импульсов, у которых степень униполярности [12]

$$
\xi=\frac{\left|\int \mathbf{E} d t\right|}{\int|\mathbf{E}| d t}
$$

близка к единице. Современное состояние исследований в области генерации предельно коротких, вплоть до аттосекундных, импульсов излучения и обзор способов получения квазиуниполярных электромагнитных импульсов представлены соответственно в [13] и [12]. Отметим, что числитель дроби в правой части (7) это модуль электрической площади импульса. Поскольку такая площадь - величина аддитивная, то эффективно и ускорение частицы последовательностью квазиуниполярных импульсов при условии сохранения у них поляризации и полярности излучения (знака электрической площади). Напомним, что приведенное рассмотрение ограничено случаем нерелятивистских скоростей частиц.

Таким образом, в данном сообщении электрическая площадь импульса сопоставлена с импульсом силы Лоренца, действующей на единичный электрический заряд при его взаимодействии с импульсом излучения. Показано соответствие закона сохранения импульса классической механики с его изменением при взаимодействии квантового объекта с интенсивным предельно коротким импульсом излучения. Подтверждена эффективность ускорения заряженных частиц квазиуниполярными импульсами излучения. Применимость результатов ограничена вкладом конечной длительности импульса и поправками к низшему приближению приближенного решения уравнения Шредингера $[8,9]$, а также для многоимпульсного режима общей длительностью излучения, которая не может превышать время жизни возбужденных уровней атома. Отметим также, что ускорение заряженной частицы должно сопровождаться электромагнитным излучением и лоренцевой силой радиационного торможения [14].

Работа поддержана грантом РФФИ № 16-02-00762_a.

\section{Список литературы}

[1] Розанов Н.Н. // Опт. и спектр. 2009. Т. 107. № 5. C. 761-765; Rosanov N.N. // Opt. Spectrosc. 2009. V. 107. N 5. P. 721.

[2] Rosanov N.N., Kozlov V.V., Wabnitz S. // Phys. Rev. A. 2010. V. 81. N 4. P. 043815 (17 pages).

[3] Розанов Н.Н. Диссипативные оптические солитоны. От микро- к нано- и атто-. М.: Физматлит, 2011. 536 с.

[4] Розанов Н.Н. // Опт. и спектр. 2015. Т. 118. № 6. C. 975-976; Rosanov N.N. // Opt. Spectrosc. 2015. V. 118. N 6. P. $943-944$.

[5] Архипов Р.М., Архипов М.В., Бабушкин И., Пахомов А.В., Розанов Н.Н. // Квантовая электроника. 2018. Т. 48. № 6. C. 532-536; Arkhipov R.M., Arkhipov M.V., Babushkin I., Pakhomov A.V., Rosanov N.N. // Quantum Electron. 2018. V. 48. N 6. P. 532-536.

[6] Розанов Н.Н., Архипов М.В., Архипов Р.М. // УФН. 2018. doi 10.3367/UFNr.2018.07.038386; Rosanov N.N., Arkhipov M.V., Arkhipov R.M. // Phys. Usp. 2018. doi 10.3367/UFNe.2018.07.038386

[7] Ландау Л.Д., Лифиии, E.M. Электродинамика сплошных сред. М.: Наука, 1982. 620 с.

[8] Розанов Н.Н. // Опт. и спектр. 2018. Т. 124. № 1. С. 75-76; Rosanov N.N. // Opt. Spectrosc. 2018. V. 124. N 1. P. 72-74.

[9] Dimitrovski D., Solov'ev E.A., Briggs J.S. // Phys. Rev. A. 2005. V. 72. P. 043411.

[10] Ландау Л.Д., Лифиии Е.М. Механика. М.: Наука, 1988. 2016 c.; Landau L.D., Lifshitz E.M. Mechanics. (ButterworthHeinemann, 1976.)

[11] Ландау Л.Д., Либиии, Е.М. Квантовая механика. Нерелятивистская теория. М.: Наука, 1989. 768 с.

[12] Архипов Р.М., Пахомов А.В., Архипов М.В., Бабушкин И., Толмачев Ю.А., Розанов Н.Н. // Письма в ЖЭТФ. Т. 105. № 6. C. 388-400 (2017); Arkhipov R.M., Pakhomov A.V., Babushkin I., Tolmachev Yu.A., Rosanov N.N. // JETP Lett. 2017. V. 105. N 6. P. 408-418.

[13] Calegari F., Sansone G., Stagira S., Vozzi C., Nisoli M. // J. Phys. B: At. Mol. Opt. Phys. 2016. V. 49. N 6. P. 062001.

[14] Ландау Л.Д., Лифшии Е.М. Теория поля. М.: Физматлит, 1988. 512 c.; Landau L.D., Lifshitz E.M. The Classical Theory of Fields (Butterworth-Heinemann, 1975). 